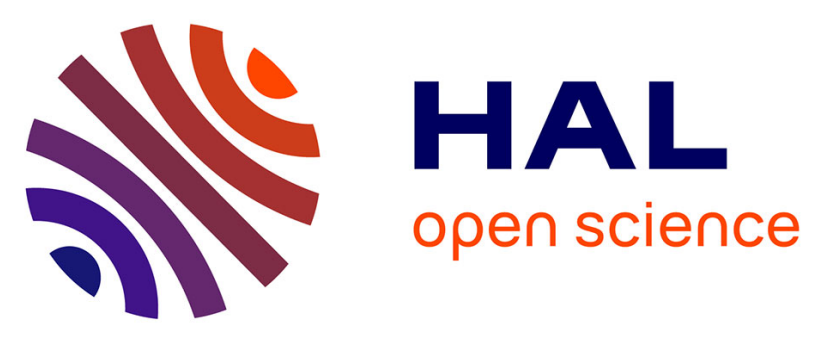

\title{
A Newton method with always feasible iterates for Nonlinear Model Predictive Control of walking in a multi-contact situation
}

Diana Serra, Camille Brasseur, Alexander Sherikov, Dimitar Dimitrov, Pierre-Brice Wieber

\section{To cite this version:}

Diana Serra, Camille Brasseur, Alexander Sherikov, Dimitar Dimitrov, Pierre-Brice Wieber. A Newton method with always feasible iterates for Nonlinear Model Predictive Control of walking in a multicontact situation. IEEE-RAS 2016 - International Conference on Humanoid Robots (Humanoids), Nov 2016, Cancun, Mexico. pp.932-937, 10.1109/HUMANOIDS.2016.7803384 · hal-01418402

\author{
HAL Id: hal-01418402 \\ https://hal.inria.fr/hal-01418402
}

Submitted on 16 Dec 2016

HAL is a multi-disciplinary open access archive for the deposit and dissemination of scientific research documents, whether they are published or not. The documents may come from teaching and research institutions in France or abroad, or from public or private research centers.
L'archive ouverte pluridisciplinaire HAL, est destinée au dépôt et à la diffusion de documents scientifiques de niveau recherche, publiés ou non, émanant des établissements d'enseignement et de recherche français ou étrangers, des laboratoires publics ou privés. 


\title{
A Newton method with always feasible iterates for Nonlinear Model Predictive Control of walking in a multi-contact situation
}

\author{
Diana Serra $^{\dagger}$, Camille Brasseur*, Alexander Sherikov*, Dimitar Dimitrov* and Pierre-Brice Wieber* \\ ${ }^{\dagger}$ DIETI, University of Naples Federico II, \\ Via Claudio 21, 80125, Naples, Italy \\ E-mail: diana.serraeunina.it \\ *INRIA Rhône-Alpes, \\ 38334 Montbonnot Cedex, France \\ E-mail: \{camille.brasseur, alexander.sherikov, pierre-brice.wieber\}@inria.fr, mail@drdv.net
}

\begin{abstract}
In this paper, we present a Nonlinear Model Predictive Control scheme, which is able to generate walking motions in multi-contact situations. Walking up and down stairs with an additional hand support is a typical example, which we address in simulation. Computing such a nonlinear control scheme is usually done with a Newton method, a potentially time-consuming procedure involving iterative linearizations. We propose here a Newton method which is specifically designed to provide at each iteration a feasible solution, always satisfying the (nonlinear) dynamic balance constraints. This results in a significant reduction in computation time, by minimizing the number of necessary iterations to reach a feasible solution.
\end{abstract}

\section{INTRODUCTION}

The main difficulty in legged locomotion is the dynamic constraints on the linear and angular momentum of the robot, due to its dependance on external contact forces to control them [1]. This is particularly evident in the Newton and Euler equations of motion of the robot, which can typically be expressed in the following form:

$$
\left[\begin{array}{c}
m(\ddot{c}+g) \\
m c \times(\ddot{c}+g)+\dot{L}
\end{array}\right] \in \mathcal{C}
$$

where $m \in \mathbb{R}$ is the mass of the robot, $c \in \mathbb{R}^{3}$ is the position of its Center of Mass (CoM) in a world frame, $L \in \mathbb{R}^{3}$ is its angular momentum with respect to the $\mathrm{CoM},-g \in \mathbb{R}^{3}$ is the acceleration due to gravity, and $\mathcal{C} \subset \mathbb{R}^{6}$ is the cone of available contact wrenches (forces and torques), which depends on the existing contacts between the robot and its environment.

The standard approach to computing, and controlling motions satisfying such contraints is through Model Predictive Control (MPC) [1], [2]. When walking on a flat, or moderately uneven ground, as long as hand contacts are not necessary, the constraint (1) can take a linear form under classical assumptions [3], and very efficient numerical methods can be employed, to compute the corresponding MPC scheme in a fraction of milli-second.

Hand contacts with the environment can be important however, to improve stability, energetic efficiency, to interact with the environment, or to simply realize otherwise infeasible motions [4], [5], [6], [7], [8]. In this case, the constraint (1) is generally nonlinear, and we have to resort to a Newton method to obtain a solution, a potentially time-consuming and uncertain procedure involving iterative linearizations.

In an MPC scheme, we have to solve a sequence of closely related optimization problems, one at each sampling time. Using tools from parametric optimization and continuation methods, it is possible to obtain very good approximate solutions to these nonlinear problems, with very few, or even only one iteration at each sampling time [9]. The problem, however, is that these approximate solutions may not always be feasible and satisfy exactly the nonlinear constraints.

This is the problem that we tackle in this paper, by proposing a Newton method based on carefully crafted linear approximate models, which ensure that the nonlinear constraint (1) is always satisfied exactly. Our approach is based on a linear approximation which is made robust to polytopic uncertainties, following the same procedure as in [3], and constraining each iteration of the Newton method to fall within the limits of the specified polytopic uncertainty. A specific form of the constraint (1) is used, where a Center of Pressure $(\mathrm{CoP})$ is introduced for foot contacts on the ground, while other external forces are considered as a generic force $f_{e} \in \mathbb{R}^{3}$ and torque $n_{e} \in \mathbb{R}^{3}$ acting on the CoM, as proposed in [8]. Simulation results show that we can very efficiently compute walking motions up and down stairs with an additional hand support, which satisfy exactly the balance constraint (1).

This paper is organized as follows. Section II presents the proposed MPC scheme for generating walking motions with additional hand support. The dynamic model of the legged robot, the kinematic and dynamic feasibility constraints for balance, and the objective function of the Optimal Control Problem (OCP) are presented. Section III presents our proposition for making sure that every iteration of our Newton scheme is always feasible, satisfying exactly the nonlinear constraint (1). Section IV finally presents an evaluation of the proposed scheme in the case of a walking motion up and down stairs, with statistics on the computational efficiency of the approach along with a comparison with other state of the art methods. 


\section{Model Predictive Control of WALKing}

\section{A. Introducing the CoP}

Let's consider the contact forces $f_{i} \in \mathbb{R}^{3}$ between the feet and the ground separately from the rest of external forces and torques in the Newton and Euler equations of motion, as proposed in [8]:

$$
\begin{aligned}
m(\ddot{c}+g) & =f_{e}+\sum_{i} f_{i}, \\
m c \times(\ddot{c}+g)+\dot{L} & =n_{e}+c \times f_{e}+\sum_{i} s_{i} \times f_{i},
\end{aligned}
$$

where $s_{i} \in \mathbb{R}^{3}$ are the positions of the contact points between the feet and the ground, and everything else is as in (1). In the following, superscripts $x, y$ and $z$ indicate the corresponding components of a vector.

In the proposed approach, dynamic feasibility can be safely checked every $100 \mathrm{~ms}$ only, so with short enough double support phases, we end up checking dynamic feasibility only during single support phases [10]. In that case, we can safely assume that every time we check dynamic feasibility, all contact points between the feet and the ground actually have the same height, $s_{i}^{z}=s^{z}$ for all $i$, even when walking up and down stairs, and gravity is orthogonal to the ground, $g^{x}=g^{y}=0$.

Dividing the Euler equation by the $z$ component of the Newton equation, we obtain

$$
\frac{m c \times(\ddot{c}+g)-c \times f_{e}-n_{e}+\dot{L}}{m\left(\ddot{c}^{z}+g^{z}\right)-f_{e}^{z}}=\frac{\sum_{i} s_{i} \times f_{i}}{\sum_{i} f_{i}^{z}} .
$$

Focusing on the $x$ and $y$ components of this equation, we can introduce the $\mathrm{CoP}$

$$
\begin{aligned}
p & =\frac{\sum_{i} s_{i}^{x y} f_{i}^{z}}{\sum_{i} f_{i}^{z}} \\
& =c^{x y}-\frac{\left(c^{z}-s^{z}\right)\left(m \ddot{c}^{x y}-f_{e}^{x y}\right)}{m\left(\ddot{c}^{z}+g^{z}\right)-f_{e}^{z}}-\frac{\Omega \tilde{n}^{x y}}{m\left(\ddot{c}^{z}+g^{z}\right)-f_{e}^{z}},
\end{aligned}
$$

where $\tilde{n}=\dot{L}-n_{e}=\sum_{i}\left(s_{i}-c\right) \times f_{i}$ is the torque exerted by the contact forces $f_{i}$ with respect to the CoM, and

$$
\Omega=\left[\begin{array}{cc}
0 & 1 \\
-1 & 0
\end{array}\right]
$$

Note that the CoP is a linear function of the $x$ and $y$ components of motion $\left(c^{x y}, \ddot{c}^{x y}, f_{e}^{x y}, \tilde{n}^{x y}\right)$, and a nonlinear function of the $z$ components $\left(c^{z}, \ddot{c}^{z}, f_{e}^{z}\right)$.

\section{B. Constraints}

Since the contact forces with the ground are usually unilateral, $f_{i}^{z} \geq 0$ for all $i$, the CoP must lie within the convex hull of the contact points $s_{i}^{x y}$ :

$$
p \in \mathcal{S}\left(s_{i}^{x y}\right) .
$$

The maximal reachable region for the CoM with respect to the center of the support foot $s_{f} \in \mathbb{R}^{3}$, is typically approximated by a convex polytope [3]:

$$
A\left(c-s_{f}\right) \leq b .
$$

Finally, external forces and torques are also bounded. We follow here the approach proposed in [7] and consider constraints:

$$
\underline{f_{e}} \leq f_{e} \leq \overline{f_{e}}, \quad \underline{\tilde{n}} \leq \tilde{n} \leq \overline{\tilde{n}} .
$$

\section{Objectives}

Our first objective is to keep the height of the CoM as close as possible to a reference $\bar{c}_{s}^{z} \in \mathbb{R}$ chosen above the kinematic limit (7),

$$
o_{1}=\left\|\left(c^{z}-s^{z}\right)-\bar{c}_{s}^{z}\right\|^{2} .
$$

Then, robustness of the walking motion to perturbations is improved by minimizing also the deviation of the CoP from the center of the support foot $s_{f}^{x y}$,

$$
o_{2}=\left\|p-s_{f}^{x y}\right\|^{2} .
$$

Finally, motion smoothness is improved by minimizing additionally the jerk of the CoM and the external wrench,

$$
o_{3}=\|\dddot{c}\|^{2}, \quad o_{4}=\left\|f_{e}\right\|^{2}, \quad o_{5}=\|\tilde{n}\|^{2} .
$$

All these are standard objectives when generating walking motions.

\section{Optimal Control Problem}

In the end, to find trajectories of the CoM with the corresponding external wrench, an OCP is formulated on a finite time horizon $T_{h}$ :

$$
\begin{array}{ll}
\underset{\dddot{c}^{*}, f_{e}, \tilde{n}}{\operatorname{minimize}} & \int_{0}^{T_{h}} \sum_{i=1}^{5} w_{i} o_{i} d t \\
\text { subject to } & (6),(7),(8), \quad \forall t,
\end{array}
$$

where $o_{i}$ are the objectives (9), (10), and (11), and $w_{i}$ are corresponding weights.

\section{Always feasible Newton ITERATES}

The following Section presents our proposition for making sure that every iteration of the Newton scheme always satisfies exactly the nonlinear constraint (1).

\section{A. Robustness to polytopic uncertainties}

Observing that equation (5) is linear with respect to the $x$ and $y$ components of motion, and nonlinear with respect to its $z$ component, walking motions were generated in [3] by adopting a Linear MPC scheme on the $x$ and $y$ components, that was made robust to the nonlinearity on the $z$ component, bounded as a polytopic uncertainty. We propose to follow a similar approach here, with the additional capacity to use hand support.

Equation (5) can be reformulated as follows,

$$
p\left(\zeta_{1}, \zeta_{2}\right)=c^{x y}-\zeta_{1}\left(\ddot{c}^{x y}-\frac{f_{e}^{x y}}{m}\right)+\zeta_{2} \Omega \tilde{n}^{x y},
$$

by introducing

$$
\zeta_{1}=\frac{m\left(c^{z}-s^{z}\right)}{m\left(\ddot{c}^{z}+g^{z}\right)-f_{e}^{z}}, \quad \zeta_{2}=\frac{1}{m\left(\ddot{c}^{z}+g^{z}\right)-f_{e}^{z}} .
$$


Note that this equation is linear with respect to $\zeta_{1}$ and $\zeta_{2}$. Let's consider now that these variables stay between some bounds:

$$
0 \leq \underline{\zeta}_{1} \leq \zeta_{1} \leq \bar{\zeta}_{1}, \quad 0 \leq \underline{\zeta}_{2} \leq \zeta_{2} \leq \bar{\zeta}_{2} .
$$

If we make sure that the constraint (6) is satisfied for all extreme values of $\zeta_{1}$ and $\zeta_{2}$, we can conclude that it is satisfied for all values in between, by a simple convexity argument:

$$
\begin{gathered}
\left\{p\left(\underline{\zeta}_{1}, \underline{\zeta}_{2}\right), p\left(\underline{\zeta}_{1}, \bar{\zeta}_{2}\right), p\left(\bar{\zeta}_{1}, \underline{\zeta}_{2}\right), p\left(\bar{\zeta}_{1}, \bar{\zeta}_{2}\right)\right\} \subset \mathcal{S}\left(s_{i}^{x y}\right) \\
\Downarrow \\
p\left(\zeta_{1}, \zeta_{2}\right) \in \mathcal{S}\left(s_{i}^{x y}\right) .
\end{gathered}
$$

Since the constraint (16) is defined with fixed, extreme values of $\zeta_{1}$ and $\zeta_{2}$, we can see from (13) that it doesn't involve anymore the $z$ components of motion, and is simply linear with respect to its $x$ and $y$ components.

On the other hand, the combination of (14) and (15) imposes linear constraints on the $z$ components of motion:

$$
\begin{aligned}
\underline{\zeta}_{1}\left(m\left(\ddot{c}^{z}+g^{z}\right)-f_{e}^{z}\right) & \leq m\left(c^{z}-s^{z}\right) \leq \bar{\zeta}_{1}\left(m\left(\ddot{c}^{z}+g^{z}\right)-f_{e}^{z}\right), \\
\bar{\zeta}_{2}^{-1} & \leq m\left(\ddot{c}^{z}+g^{z}\right)-f_{e}^{z} \leq \underline{\zeta}_{2}^{-1}
\end{aligned}
$$

This way, if we make sure that the $x$ and $y$ components of motion satisfy the linear constraint (16) while the $z$ components satisfy the linear constraints (17) and (18), we can conclude that the 3D motion satisfies the nonlinear constraint (6).

\section{B. Constraining Newton iterates}

Each iteration of a Newton scheme involves computing the solution to a linear approximation of the nonlinear problem. The key to our approach is to constrain each of these iterations, to satisfy the linear constraints (16), (17) and (18), in order to make sure that the nonlinear constraint (6) is always satisfied exactly.

Instead of considering fixed bounds on $\zeta_{1}$ and $\zeta_{2}$ in (15), that would require to be finely tuned as in [3], we propose here to adapt them at each iteration in the following way, based on the value of the parameters $\zeta_{1}$ and $\zeta_{2}$ at the previous iteration:

$$
\begin{array}{ll}
\underline{\zeta}_{1}^{(j)}=\zeta_{1}^{(j-1)}-k \mu_{1}, & \bar{\zeta}_{1}^{(j)}=\zeta_{1}^{(j-1)}+k \mu_{1}, \\
\underline{\zeta}_{2}^{(j)}=\zeta_{2}^{(j-1)}-k \mu_{2}, & \bar{\zeta}_{2}^{(j)}=\zeta_{2}^{(j-1)}+k \mu_{2},
\end{array}
$$

with positive constants $k, \mu_{1}$ and $\mu_{2}$.

\section{Simulation Results}

The proposed approach is evaluated on a walking motion up and down stairs, with an external force generated by hand supports at two predefined time intervals, as shown on Figure 1. This motion is generated for a simulated HRP4 [11], using the parameters provided in Table I.

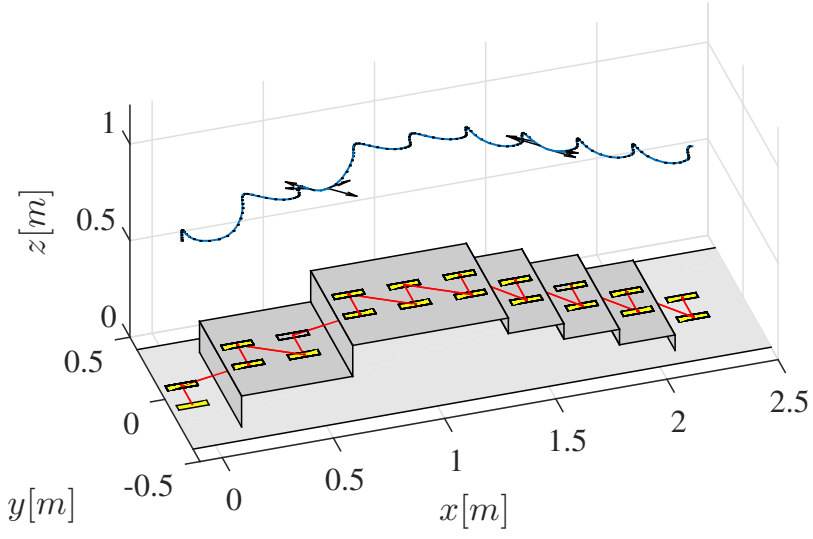

Fig. 1. An example of walking motion climbing up and down stairs, generated online with the proposed Nonlinear MPC scheme. Footprints are represented with yellow rectangles, while their sequence is indicated with a red broken line. The trajectory of the CoM is indicated in blue, and the corresponding external force appears as black arrows.

TABLE I

SIMULATION PARAMETERS

\begin{tabular}{|c|c|}
\hline Sampling period & $0.1 \mathrm{~s}$ \\
\hline Length of the MPC horizon & $1.6 \mathrm{~s}$ \\
\hline Target height for the CoM & $\bar{c}_{s}^{z}=0.9 \mathrm{~m}$ \\
\hline Mass of the robot & $m=37.84 \mathrm{~kg}$ \\
\hline Size of the feet & $3.6 \times 13.6 \mathrm{~cm}^{2}$ \\
\hline Bounds on $f_{e}$ & $\underline{f_{e}}=-25 \mathrm{~N}, \overline{e_{e}}=25 \mathrm{~N}$ \\
\hline Bounds on $\tilde{n}$ & $\underline{\tilde{n}}=-25 \mathrm{Nm}, \tilde{\tilde{n}}=25 \mathrm{Nm}$ \\
\hline Polytopic uncertainty & $\mu_{1}=0.01 \mathrm{~s}^{2}, \mu_{2}=0.0005 \mathrm{~N}^{-1}$ \\
\hline
\end{tabular}

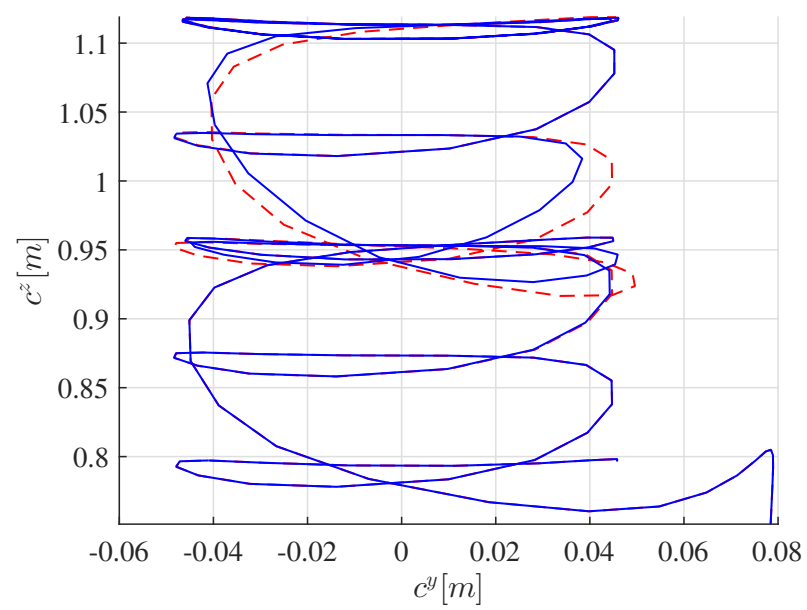

Fig. 2. Comparison between CoM trajectories in the frontal plane $(y, z)$ with (blue line) and without (dashed red line) additional hand support. 

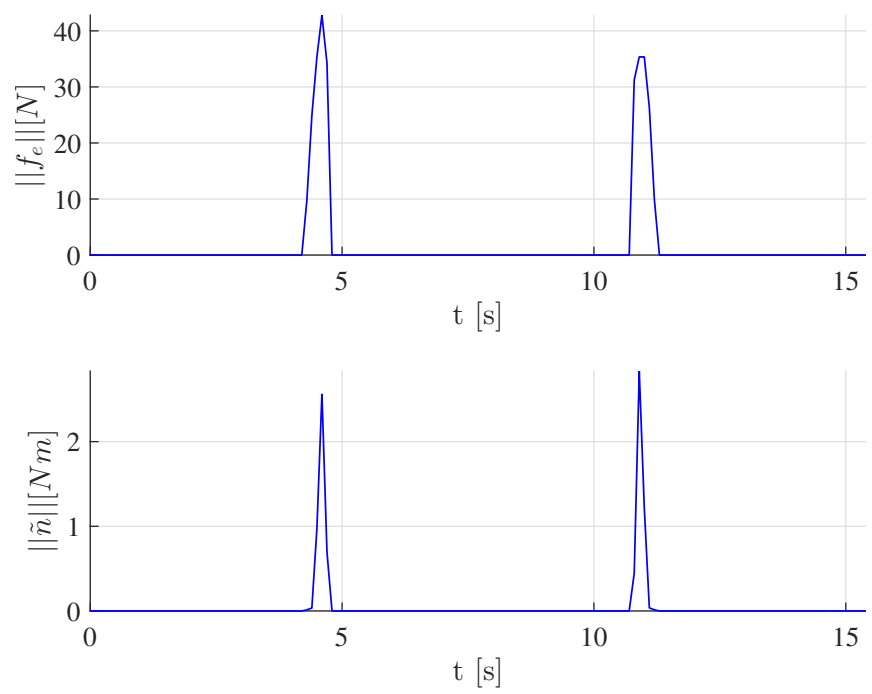

Fig. 3. Evolution of the norm of the external force $f_{e}$ (top) and torque $\tilde{n}$ (bottom).

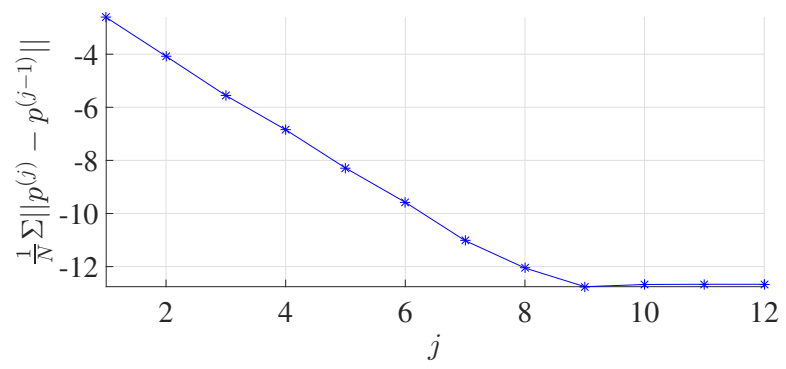

Fig. 4. Semi-log graph showing the convergence of the CoP trajectory over iterations of the Newton scheme.

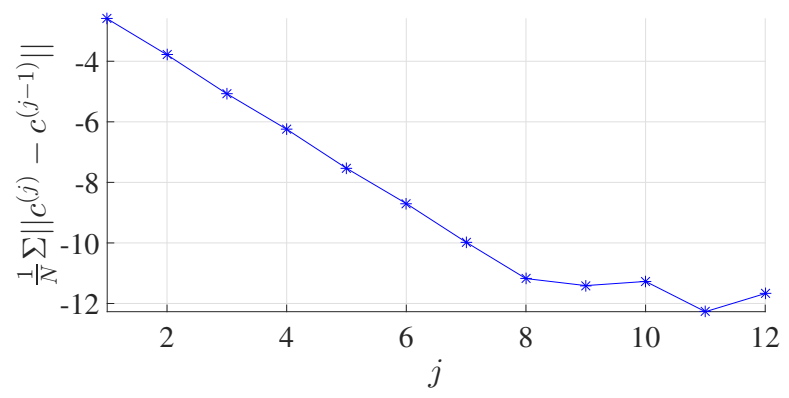

Fig. 5. Semi-log graph showing the convergence of the CoM trajectory over iterations of the Newton scheme.

TABLE II

WEIGHTS FOR THE DIFFERENT OBJECTIVES

\begin{tabular}{|c|c|}
\hline Distance between $c^{z}$ and its reference & $w_{1}=1$ \\
\hline Distance between $p$ and its reference & $w_{2}=1$ \\
\hline CoM jerk norm & $w_{3}=1.5 * 10^{-4}$ \\
\hline External force norm & $w_{4}=10^{-7}$ \\
\hline External torque norm & $w_{5}=10^{-4}$ \\
\hline
\end{tabular}

\section{A. Generated motion}

We can see in Figure 2 how the presence of an additional hand support during the steps up and down stairs affects the motion of the CoM in the frontal plane $(y, z)$. The weighted sum of objectives $o_{1}, o_{2}$ and $o_{3}$ naturally decreases when the external wrench is employed, since it represents an additional degree of freedom for the OCP. Figure 3 shows the corresponding evolution of the norm of the external force $f_{e}$ and torque $\tilde{n}$ during the two predefined moments. Since a minimization of the norm of the wrench is performed, higher weights, $w_{4}$ and $w_{5}$, on $f_{e}$ and $\tilde{n}$ generate lower values of the resultant wrench. We use a higher weight for the external torque $\left(w_{5}>w_{4}\right)$ in order to reduce the rotational motion around the CoM. The proposed weights $w_{i}$ are given in Table II.

We can see in Figures 4 and 5 how the CoP and the CoM converge over iterations of the Newton scheme for a given preview horizon. We can observe that the first iteration already provides a very good approximate solution, only a few millimeters away from the optimum. More importantly, the method we propose makes sure that it satisfies exactly the nonlinear constraint (6).

\section{B. Nonlinear constraint and polytopic uncertainty}

Figure 6 shows how the $\mathrm{CoP}$ always stays inside the quadrilateral defined by the four extreme points $p\left(\underline{\zeta}_{1}, \underline{\zeta}_{2}\right)$, $p\left(\underline{\zeta}_{1}, \bar{\zeta}_{2}\right), p\left(\bar{\zeta}_{1}, \underline{\zeta}_{2}\right)$ and $p\left(\bar{\zeta}_{1}, \bar{\zeta}_{2}\right)$ due to argument $(16)$, while all the extreme points are kept inside the support polygon $\mathcal{S}\left(s_{i}^{x y}\right)$ by the corresponding constraints. Four different computations are shown, with a range of polytopic uncertainty $\left\{\mu_{1}, \mu_{2}\right\}$ multiplied by four different coefficients, $k \in\{1,0.6,0.4,0.2\}$. We can see how the choice of a larger range leads to much more conservative constraints. Figure 7 shows how this more conservative constraint affects the value that can be reached for the objective function. It appears clearly that a smaller range leads to better results.

Note that the range of values for the polytopic uncertainties was chosen empirically, as inappropriate values can quickly lead to infeasible problems, what would require a relaxation of constraints.

\section{Computational efficiency}

Solutions are computed with qpOASES [12]. Without using hotstart, on a relatively slow Intel Core i5-4200U @ $1.60 \mathrm{GHz}$, the solutions are obtained in approximately $8 \mathrm{~ms}$ (average time over the whole trajectory generation). This result can certainly be improved significantly with hotstart. As a comparison, the computation time reported for a similar problem in [13] is $5.5 \mathrm{~s}$ for 85 iterations, which gives approximately $65 \mathrm{~ms}$ for each iteration, on a much faster CPU. Not only is our Newton scheme much safer, by providing iterates which are always feasible, it appears also to be an order of magnitude more efficient in terms of computation time. 

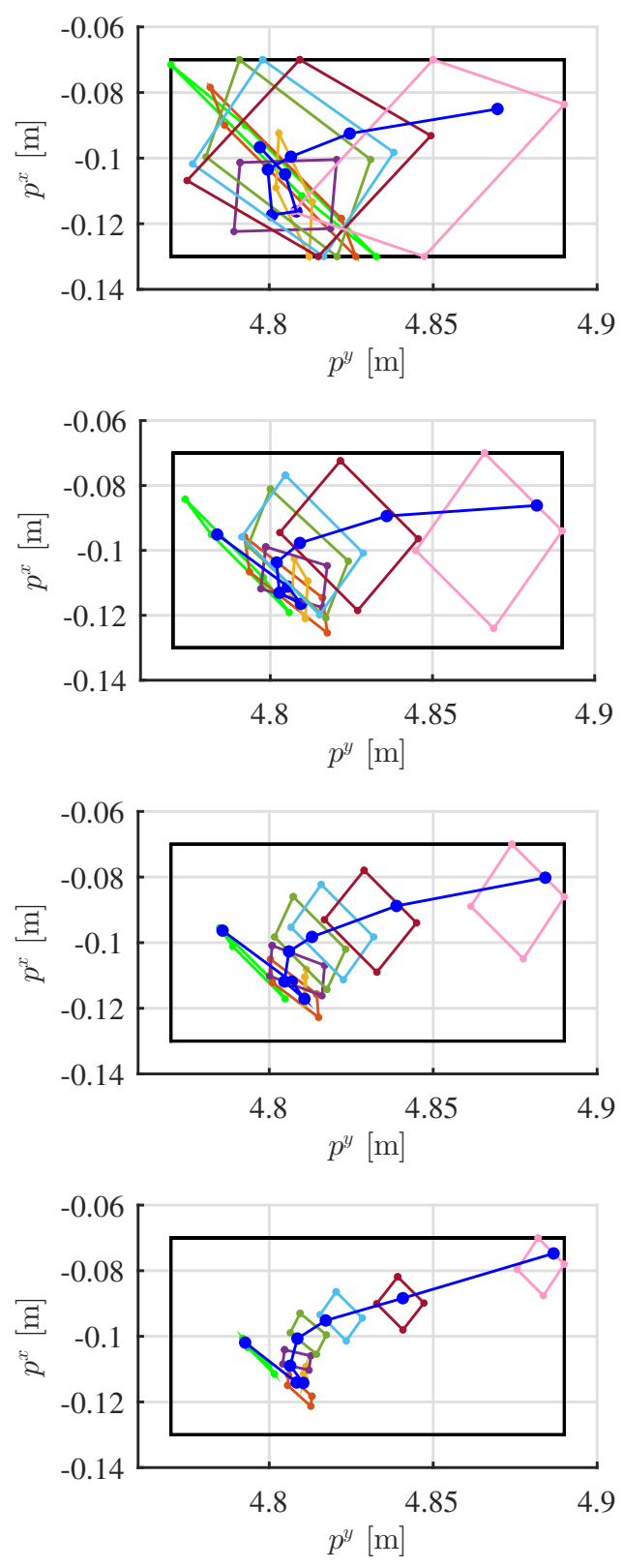

Fig. 6. The trajectory of the $\mathrm{CoP}$ (blue line) is always kept inside the quadrilateral defined by the four extreme points $p\left(\underline{\zeta}_{1}, \underline{\zeta}_{2}\right), p\left(\underline{\zeta}_{1}, \bar{\zeta}_{2}\right)$, $p\left(\bar{\zeta}_{1}, \underline{\zeta}_{2}\right)$ and $p\left(\bar{\zeta}_{1}, \bar{\zeta}_{2}\right)$, which are all kept inside the support polygon $\mathcal{S}\left(s_{i}^{x y}\right)$ (black rectangle). The range of polytopic uncertainty $\mu_{1}$ and $\mu_{2}$ is multiplied here by four different coefficients, from top to bottom: $k=1$, $k=0.6, k=0.4, k=0.2$.

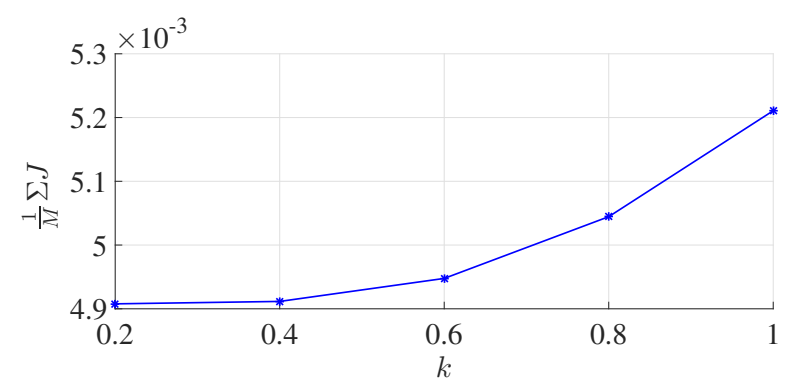

Fig. 7. Mean value of the objective function over the whole motion w.r.t. the coefficient $k$. The objective function is impacted by the choice of the coefficient $k$, by as much as $6 \%$.

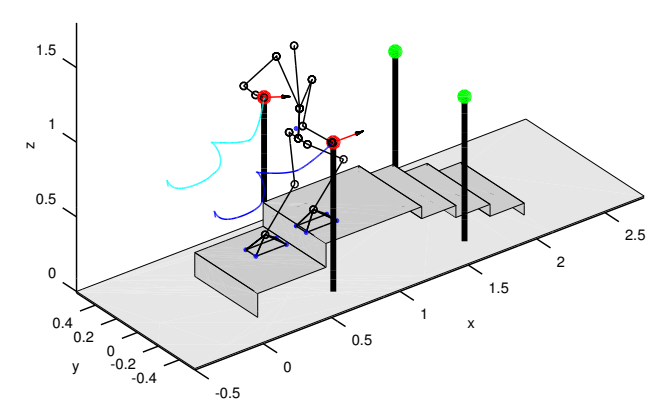

Fig. 8. Whole body motion with two hand contacts to help climb up a $16 \mathrm{~cm}$ high step. Blue and cyan curves indicate trajectories of the right and left hands respectively. Green and red dots correspond to inactive and active contact points. Arrows represent normalized contact forces at the contact points.

\section{Whole body motion}

Simulations of the resulting whole body motion are performed (see Figure 8), using a standard inverse dynamics approach [14], [15]. Our whole body motion controller employs PD controllers to track the reference trajectory of the CoM produced by the MPC scheme, and the trajectories of the feet and hands generated using cubic polynomials. Positions of the hands are controlled only during the short intervals when they are approaching the contact points - during the rest of the simulation the reference configuration of the arms is maintained with PD controllers instead. The whole body motion controller obeys contact friction constraints and constraints due to dynamics and kinematics of the robot. These constraints include the joint torque limits, which, however, are never reached in our simulations.

These simulations are illustrated in the accompanying video [16], which consists of four parts: the first two illustrate walking on a flat ground, while the last two demonstrate walking up and down stairs. Due to the fact that the reference external wrench acts directly on the CoM, we have a relative freedom in choosing the contact configurations. In order to 
demonstrate this we generate the reference external wrench using a single hand contact in the first and third parts of the video, while in the second and fourth parts we produce exactly the same external wrenches using two hand contacts.

In the case of walking on a flat ground, tuning of the MPC and whole body motion controller amounted to finding kinematically feasible contact point positions, which can be relatively easily parameterized with the CoM position. We experienced more difficulties while working on walking on stairs. Most of them were related to infeasibility of the reference trajectories due to the mechanical limits of the knee (going up) and ankle (going down) joints. The latter appears to be a particularly severe limitation, which makes walking downstairs with flat feet a much more challenging task than going upstairs. We alleviated the issues with the joint limits by tuning the swing foot trajectories and the constraint on the maximal distance between the feet and the CoM. In addition, we considered relatively low stairs when going downstairs $(8 \mathrm{~cm})$, instead of much higher ones when going upstairs $(16 \mathrm{~cm})$.

\section{CONCLUSION}

We have proposed a Newton method which provides feasible iterates, always satisfying the nonlinear constraint (6), for a Nonlinear MPC scheme generating walking motions in multi-contact situations. It is based on a linear approximation which is made robust to polytopic uncertainties, constraining each iteration of the Newton method to fall within the limits of these polytopic uncertainties. The simulation of a walking motion up and down stairs with additional hand support demonstrates the applicability of our method, with remarkable computational efficiency. This approach works as well for different multi-contact situations, since the generated external wrench is not dependent on the specific contact configuration.

The proposed method relies on predefined footsteps and hand supports. Automatic footstep placement on horizontal surfaces could be included easily using the methods introduced in [10], but more complex cases of uneven ground and multi-contact situations require much more advanced planning methods [6], [17], [18], [19]. Our next steps however are to validate the proposed approach on a real robot, and to evaluate how a lexicographic approach could be introduced, to automatically decide when it is necessary to use the additional hand support, as in [20].

\section{ACKNOWLEDGMENTS}

This work has been funded by the PSPC Romeo 2 project, the EU H2020 Comanoid Research and Innovation Action (RIA) and the RoDyMan project, which has received funding from the European Research Council FP7 Ideas under Advanced Grant agreement number 320992.

\section{REFERENCES}

[1] P. B. Wieber, R. Tedrake, and S. Kuindersma, "Modeling and control of legged robots," in The hadbook of robotics, Springer, Ed., 2015, ch. 48 .
[2] D. Q. Mayne, J. B. Rawlings, C. V. Rao, and P. O. M. Scokaert, "Constrained model predictive control: Stability and optimality," $\mathrm{Au}$ tomatica, vol. 36, no. 6, pp. 789-814, 2000.

[3] C. Brasseur, A. Sherikov, C. Collette, D. Dimitrov, and P. B. Wieber, "A robust linear MPC approach to online generation of 3D biped walking motion," in IEEE-RAS 15th International Conference on Humanoid Robots. IEEE, 2015, pp. 595-601.

[4] K. Harada, H. Hirukawa, F. Kanehiro, K. Fujiwara, K. Kaneko, S. Kajita, and M. Nakamura, "Dynamical balance of a humanoid robot grasping an environment," in International Conference on Intelligent Robots and Systems, vol. 2, 2004, pp. 1167-1173.

[5] K. Koyanagi, H. Hirukawa, S. Hattori, M. Morisawa, S. Nakaoka, K. Harada, and S. Kajita, "A pattern generator of humanoid robots walking on a rough terrain using a handrail," in IEEE/RSJ International Conference on Intelligent Robots and Systems. IEEE, 2008, pp. 2617-2622.

[6] H. Audren, J. Vaillant, A. Kheddar, A. Escande, K. Kaneko, and E. Yoshida, "Model preview control in multi-contact motionapplication to a humanoid robot," in IEEE/RSJ International Conference on Intelligent Robots and Systems. IEEE, 2014, pp. 4030-4035.

[7] M. Kudruss, M. Naveau, O. Stasse, N. Mansard, C. Kirches, P. Souères, and K. Mombaur, "Optimal control for whole-body motion generation using center-of-mass dynamics for predefined multi-contact configurations," in IEEE-RAS 15th International Conference on $\mathrm{Hu}$ manoid Robots. IEEE, 2015, pp. 684-689.

[8] D. J. Agravante, A. Cherubini, A. Sherikov, P. B. Wieber, and A. Kheddar, "Human-Humanoid Collaborative Carrying," 2016, working paper or preprint. [Online]. Available: http://hal-lirmm.ccsd. cnrs.fr/lirmm-01311154

[9] M. Diehl, "Real-time optimization for large scale nonlinear processes," Ph.D. dissertation, 2001.

[10] A. Herdt, H. Diedam, P. B. Wieber, D. Dimitrov, K. Mombaur, and M. Diehl, "Online walking motion generation with automatic footstep placement,” Advanced Robotics, vol. 24, no. 5-6, pp. 719-737, 2010.

[11] K. Kaneko, F. Kanehiro, M. Morisawa, K. Akachi, G. Miyamori, A. Hayashi, and N. Kanehira, "Humanoid robot hrp-4 - humanoid robotics platform with lightweight and slim body," in IEEE/RSJ International Conference on Intelligent Robots and Systems, Sept 2011, pp. 4400-4407.

[12] H. J. Ferreau, C. Kirches, A. Potschka, H. G. Bock, and M. Diehl, "qpOASES: A parametric active-set algorithm for quadratic programming," Mathematical Programming Computation, vol. 6, no. 4, pp. 327-363, 2014.

[13] J. Carpentier, S. Tonneau, M. Naveau, O. Stasse, and N. Mansard, "A versatile and efficient pattern generator for generalized legged locomotion," in IEEE International Conference on Robotics and Automation. IEEE, 2016, pp. 3555-3561.

[14] Y. Fujimoto and A. Kawamura, "Proposal of biped walking control based on robust hybrid position/force control," in IEEE International Conference on Robotics and Automation, vol. 3, Apr 1996, pp. 2724 2730 vol.3.

[15] L. Saab, O. E. Ramos, F. Keith, N. Mansard, P. Souères, and J. Y. Fourquet, "Dynamic whole-body motion generation under rigid contacts and other unilateral constraints," IEEE Transactions on Robotics, vol. 29, no. 2, pp. 346-362, 2013.

[16] [Online]. Available: https://youtu.be/NSDiSRPnxZ0

[17] R. Deits and R. Tedrake, "Footstep planning on uneven terrain with mixed-integer convex optimization," in IEEE-RAS International Conference on Humanoid Robots. IEEE, 2014, pp. 279-286.

[18] A. Escande, A. Kheddar, and S. Miossec, "Planning contact points for humanoid robots," Robotics and Autonomous Systems, vol. 61, no. 5, pp. 428-442, 2013.

[19] S. Tonneau, N. Mansard, C. Park, D. Manocha, F. Multon, and J. Pettré, "A reachability-based planner for sequences of acyclic contacts in cluttered environments," in International Symposium on Robotics Research, 2015.

[20] A. Sherikov, D. Dimitrov, and P. B. Wieber, "Balancing a humanoid robot with a prioritized contact force distribution," in IEEE-RAS 15th International Conference on Humanoid Robots, Nov 2015, pp. 223228 . 